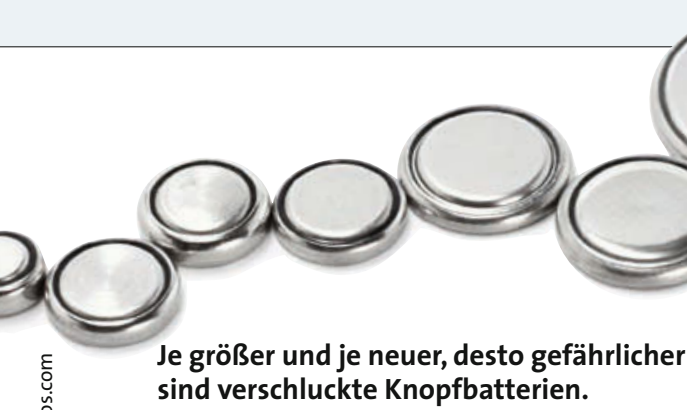

keit zu einem Schleimhautschaden führten als gebrauchte. Merken sollte man sich, dass bereits innerhalb von zwei Stunden schwere Verätzungen nachgewiesen wurden.

Die Ergebnisse der Analyse führen zu neuen Empfehlungen (mit einem guten Flussdiagramm zum Vorgehen in der Originalpublikation), die sicher auch in Europa Beachtung finden sollten:

- Jede Knopfbatterieingestion ist ein Notfall, der rasches Handeln erfordert.

- Die erste Maßnahme ist eine RöntgenThorax-Übersicht mit Halsdarstellung.

- Batterien im Ösophagus müssen innerhalb von zwei Stunden entfernt werden, unabhängig davon, ob der Patient nüchtern ist oder nicht.
_-Falls die Batterie sich bereits im Magen befindet, kann in den meisten Fällen abgewartet werden. Ausnahme: Ein Magnet ist gleichzeitig verschluckt worden. Die Toxizität der Chemikalien spielt keine große Rolle mehr.

- Die Ösophagoskopie und endoskopische Entfernung der Batterie ist Methode der Wahl. Dabei muss die Ausdehnung, Tiefe und Lokalisation des Gewebeschadens festgehalten werden. Es sollte auch die Position des negativen Pols der Batterie dokumentiert werden, da hier das Ausmaß der lokalen Schäden am größten ist. Auch nach erfolgreicher Entfernung sind Spätkomplikationen nach Tagen bis Wochen möglich.

- Bei Kleinkindern mit unklaren Schluckproblemen, Erbrechen sowie plötzlich eingetretenen Atemwegskomplikationen muss auch an eine Batterieingestion gedacht werden.

Ein weiterer Artikel in der gleichen Ausgabe von Pediatrics [Litovitz T. et al. Pediatrics 2010; 125: 1178-83] untersucht anhand von 8.000 Fällen, woher die ver- schluckte Knopfbatterie kam. In 60\% der Fälle wurden die Batterie von den Kindern selbst aus dem jeweiligen Gerät entfernt. Zur Prävention wäre es nötig, die Geräte so zu verbessern, dass eine Entfernung ohne Hilfsmittel nicht möglich ist.

Kommentar: Knopfbatterieingestionen mit Verbleib der Zellen im Ösophagus sind insgesamt selten, sollten aber wegen des zunehmenden Risikos schwerer Komplikationen ernst genommen werden. Beim Verdacht muss rasch und gezielt untersucht und behandelt werden. Die endoskopische Entfernung aus dem Ösophagus mit Dokumentation des bereits eingetretenen Schleimhautschadens ist die Methode der Wahl. Auf die Bedeutung der 20-mm-Lithiumzellen mit 3 Volt muss besonders geachtet werden. Die veränderten Empfehlungen zum Vorgehen sollten berücksichtigt werden.

\section{Dr. Martin Claßen}

Litovitz T et al. Emerging battery-ingestion hazard: clinical implications. Pediatrics 2010; 125: 1168-77

\title{
Beidhänder im Nachteil
}

\section{Sprachdefizite sowie Symptome einer ADHS sind häufig mit Schul- problemen verbunden, die möglichst früh erkannt werden sollten. Wissenschaftler untersuchten nun, ob Beidhänder vermehrt mit diesen Problemen zu kämpfen haben.}

E ür ür die Studie aus Finnland wurden prospektive Daten der „Northern Finland Birth Cohort 1986“ ausgewertet, die seit 1986 Daten von insgesamt 7.871 Kindern von Geburt bis zum Alter von 16 Jahren erfasst. Kinder, die noch im Alter von fünf Jahren beidhändig waren, wurden hinsichtlich Sprachentwicklung und ADHS-Symptomatik mit Kindern, die zu diesem Zeitpunkt rechts- bzw. linkshändig waren, verglichen. Zur Erhebung der Daten wurden Eltern-, Lehrerund Eigenbeurteilungen der Jugendlichen herangezogen, die Aussagen über Sprachschwierigkeiten, schulische Leistungen und mentale Probleme inklusive mögliche Symptome einer ADHS machten.

Es zeigte sich, dass Kinder, die im Alter von acht Jahren beide Hände benutzten, ein zweifach erhöhtes Risiko für Sprach- probleme und Lernschwierigkeiten im Vergleich zu Rechtshändern hatten. Im Alter von 16 Jahren war immer noch ein erhöhtes Risiko für Sprach- und Lernprobleme, zusätzlich zu einem erhöhten Risiko für psychische Probleme incl. ADHS vorhanden.

Kommentar: Das Persistieren von Beidhändigkeit wird als eine Störung in der zerebralen Lateralisierung gedeutet. Die Kausalität zwischen Beidhändigkeit und dem Auftreten von Sprech- und Lernstörungen sowie von ADHS bleibt unklar. Es liegt jedoch die Vermutung nahe, dass eine zerebrale Maturationsverzögerung/ -störung sowohl zu einer verzögerten Händigkeit als auch zu Sprach- bzw. Lernstörungen und ADHS führen kann. Eine persistierende Beidhändigkeit bei

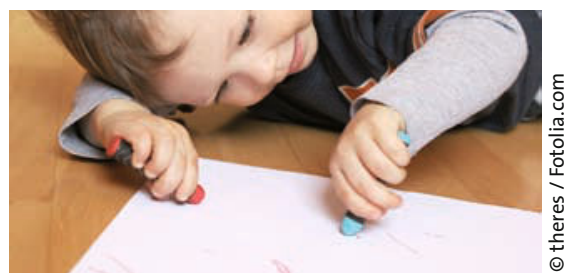

Kinder, die weder links- noch rechtshändig sind, haben ein höheres Risiko für Sprachschwierigkeiten.

Kindern im Alter von fünf Jahren sollte daher zu einer sorgfältigen Beobachtung hinsichtlich Schulperformance und dem Auftreten einer ADHS veranlassen. Es gibt jedoch keine Hinweise darauf, dass ein „Training der Händigkeit“, z. B. durch kinesiologische Übungen, das Auftreten von Lernstörungen bzw. ADHS verhindern kann. Ebenso wenig ist zu erwarten, dass eine direkte kausale Beziehung zwischen Händigkeit und Lernstörung/ ADHS besteht. Dr. Kirsten Stollhoff

Rodriguez A et al. Mixed-handedness is linked to mental health problems in children and adolescents. Pediatrics 2010; 125: e340-8 